\title{
Automatic detection of objective prism stellar spectra
}

\author{
E. Bratsolis ${ }^{1,2}$, I. Bellas-Velidis ${ }^{1}$, E. Kontizas ${ }^{1}$, F. Pasian ${ }^{3}$, A. Dapergolas ${ }^{1}$, and R. Smareglia ${ }^{3}$ \\ 1 Astronomical Institute, National Observatory of Athens, P.O. Box 20048, GR-11810 Athens, Greece \\ 2 Section of Astrophysics, Astronomy and Mechanics, Department of Physics, University of Athens, GR-15784 Athens, Greece \\ 3 Osservatorio Astronomico di Trieste, Via G.B. Tiepolo 11, I-34131 Trieste, Italy
}

Received March 16; accepted April 20, 1998

\begin{abstract}
Object detection is a common problem in image processing and particularly in astronomy and astrophysics, when a huge number of objects are to be processed. Such is the case of objective prism plates, where thousands of spectra are recorded. We present a new method for automatic detection of stellar spectra on digitally-scanned objective prism plates. Our method is based on signal processing techniques with emphasis on obtaining the maximum signal-to-noise ratio from the data, as well as on applying a number of morphological criteria of one-dimensional stellar spectra images. It uses only the prism plate image without the need of the corresponding direct plate. This fully automated method gives inpressive results in the detection of stellar spectra.
\end{abstract}

Key words: methods: data analysis; image processing stars: objective prism spectra

\section{Introduction}

The analysis of the distribution of spectral types in stellar systems is a powerful diagnostic for the estimation of their composition, age and evolutionary stage. The observing material mainly used for this task are prism spectral plates taken with Schmidt-class telescopes. Such a plate generally contains thousands of spectra, and there are prism-plate libraries and digitized data bases in several astronomical centers that can be exploited for this analysis. To deal with the pool of spectral data and to fully exploit them, highly automated image analysis tools need to be developed.

Extracting the physical quantities from the digitized spectral plates involves three main stages: detection of the spectra, extraction of their images, and classification of the spectra. The purpose of this paper is to present a

Send offprint requests to: E. Bratsolis (ebrats@atlas.uoa.gr) new, fully automated method for the detection of spectra. Previous works for automated classification of stellar spectra (von Hippel et al. 1994), for quasar spectral analysis (Hewett et al. 1995), for galaxy classification (Lahav et al. 1996) or for galaxy redshift measurements (Tucholke \& Schuecker 1992; Schuecker 1996) applied spectra detection using coordinates of the corresponding stars either determined for the purpose with the Automated Plate Measuring (APM) system or taken from catalogues (e.g. GSC). Our method is based on processing only the digitized prism plates without the need of the corresponding direct plates, necessary for the above-mentioned methods.

For this study, high-quality film copies of IIIa-J plates taken with the $1.2 \mathrm{~m}$ UK Schmidt Telescope in Australia have been used. The spectral plates are with dispersion of $830 \AA / \mathrm{mm}$ at $\mathrm{H}_{\gamma}$ and spectral range from 3400 to $5000 \AA$. The magnitude limit is about 16.5 in $V$. The photographic material has been digitized at the Trieste Observatory by means of a $P D S 1010 \mathrm{~A}$ microdensitometer and at the Royal Observatory of Edinburgh using the Super-COSMOS measuring machine. The spectra detection method has been developed under MIDAS (Midas 96NOV 1996) as a part of the objective prism plate image processing context OBJPR (Pasian et al. 1997).

The new algorithm, based on signal processing methods, is presented in Sect. 2, and its implementation is described in Sect. 3. Results from the tests of the new method are given in Sect. 4 .

\section{The signal processing based method DETSP}

Various automatic detection methods such as binarization and thinning, Sobel filters and logical feature filters give incomplete results so far (Smareglia et al. 1994). Our approach is based on signal processing methods (Bratsolis 1997). 


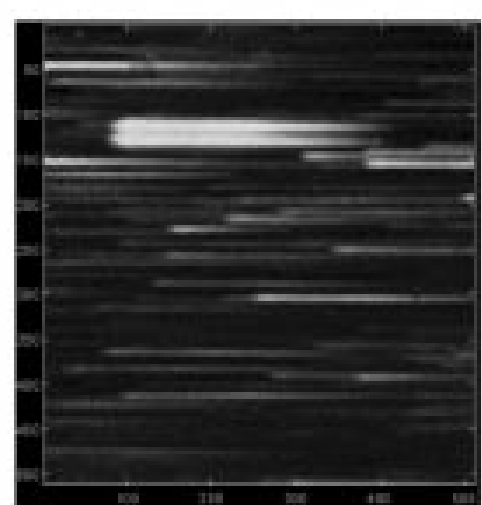

a.

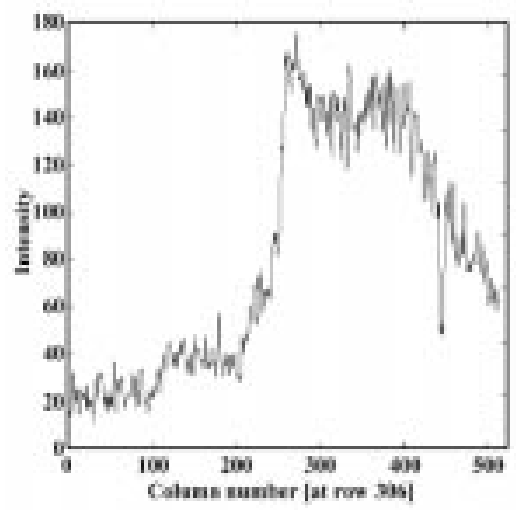

d.

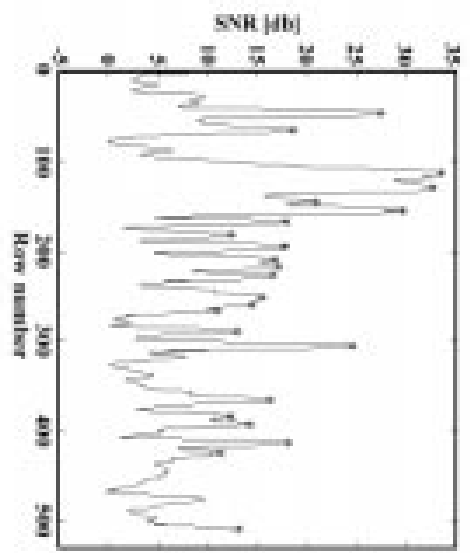

b.

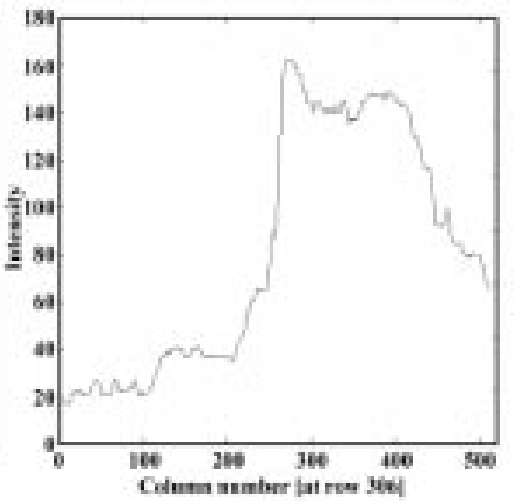

e.

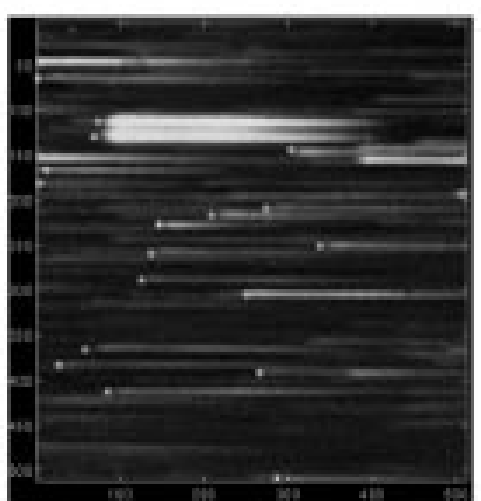

c.

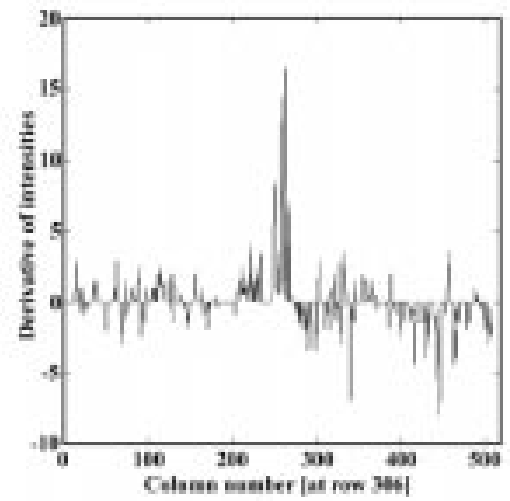

f.

Fig. 1. a) Initial image $512 \times 512$; b) A peak picking to separate the stellar spectrum rows from spurious peaks; c) Final results of position determination; d) The row 306 as a one-dimensional signal; e) The row 306 after a median and an average filter; f) Derivative results of filtered row 306

\subsection{The detection algorithm}

In the case of images obtained from objective prism plates, the objects to analyze (the stellar spectra), although possessing a great variety of internal structural characteristics, show some evident common features. For the detection of a spectrum, the most important feature is its beginning that is characterized by the presence of intense gradients. So, the main problem of the detection is reduced to the determination of the beginning of the spectrum.

Let us consider an objective prism image (Fig. 1a). It contains spectra of bright and faint stars, single or overlapped, and a random noise. This is a part of the digitized spectral plate, a subimage of $N \times M$ pixels. Let $I(x, y)$ denote the value of the pixel $(x, y)$ where the $x$-axis is down the dispersion. The subimage itself can be considered as a set of $M$ discrete one-dimensional signals $I_{y}(x)=I(x, y)$, where $M$ is the number of rows and every signal is an independent row $y$. The energy $E_{y}$ of a particular onedimensional signal is defined by Eq. (1).

$E_{y}=\sum_{x=1}^{N} I_{y}^{2}(x)$
The row with the minimum energy $\min \left(E_{y}\right)$ is considered as representing a background. Now we can use the definition of the variation of energy for every row in decibels $S N R_{y}$, see Eq. (2). Analyzing this variation with an appropriate peak-picking algorithm, we can identify energy peaks corresponding to stellar spectrum rows and separate them from spurious peaks (crossed peaks in Fig. 1b).

$S N R_{y}=20 \log _{10}\left(\frac{E_{y}}{\min \left(E_{y}\right)}\right)$.

The detection of such peaks (rows) is the first step of the algorithm. The second is to fix in each such row the beginning of the spectrum image. Let us consider now the onedimensional signal of row 306 (Fig. 1d). The grand "gap" on the right could be a defect (here) or a real absorption line. The aim of position determination is to detect the region before the great change from background on the left (Fig. 1d).

We find it by taking the derivatives and choosing the maximal. In advance, we apply to the signal an appropriate composition of median and average filters resulting in an optimally-smoothed spectrum scan (Fig. 1e) with its derivative shown in Fig. 1f. The latter is used to determine the very beginning of the spectrum. We overcome the 
problem of absorption lines by considering the maximal derivative in every case at a point which is leftward from the maximum value of the row. The final results of spectra detection on this subimage are illustrated in Fig. 1c.

\subsection{The image sampling}

It can easily be seen that the sizes of the subimage, especially down the wavelength direction, is of crucial importance for the algorithm just described. To avoid missing two spectra on the same row, the subimage should be shorter than the spectrum length. Fixing the subimage length, we have to put some restriction on its width, to take into account the local signal-to-noise ratio that gives the best detection limits for the peak-picking process. Both these imply that an image frame should be handled in pieces, subframes.

Taking a subframe from an image, there are often spectra that begin near the subframe's left or right end or are crossed by the bottom or top side. Such spectra probably could not be detected. To avoid this, a partial overlapping of the subframes should be applied in both axes. Having in mind the spectral image parameters, it is optimal to set the subframe sizes to a value about the half of the spectral length, whereas the overlapping size to about twice of the spectral width.

\section{The spectrum detection procedure DETSP}

The above-described algorithm is implemented in a procedure named DETSP, part of a general context OBJPR (Pasian et al. 1997) for objective prism image processing, developed in collaboration by the Astronomical Observatory of Trieste and the Astronomical Institute of the National Observatory of Athens. The context is worked out under the Munich Image Data Analysis System (MIDAS 96NOV 1996).

The DETSP takes as input an image frame from a digitized spectral plate and particular parameters for the spectral images (dispersion, length, width). The subframe overlapping can be applied to both axes (defaulted), only down the wavelength axis, or skipped altogether. Optionally, working image displaying and/or online graphics can be switched on. Output is a MIDAS format table with the detected spectral images positions. It is suitable for use by the procedures of the OBJPR, say for extraction the detected spectral images. The processing is carried out in four sequential stages:

a. Image frame preprocessing

The whole image frame is filtered by a sequence of median and smoothing filters. A grid of subframes (see Sect. 2.2) is fixed on the filtered image, according to the overlapping mode.

\section{b. Subframe signal processing}

Each one of the fixed subframes is processed applying the signal detection algorithm (see Sect. 2.1). The detected spectral positions are saved in table.

c. Detection table processing

There are possible double detections of spectra near the edges of neighboring subframes. For this reason, the table of detected spectra is now processed to remove the doubling. It is sorted as well.

d. Detection fine adjustment

The signal processing approach is used again. Now, as many subframes are fixed as the number of detected spectra. The subframes are narrower and each one includes a particular detected spectrum image. This leads to fine adjustment of the position. The adjusted position table is finally sorted.

\section{Results}

The DETSP procedure tests have been run in the Astronomical Institute under MIDAS-96NOV environment on a Hewlett-Packard HP-C160 workstation with 64-bit CPU, 64MB RAM, and operating system HP-UX 10.20. We used two frames from digitized high-quality copies of spectral plates taken with the $1.2 \mathrm{UK}$ Schmidt Telescope in Australia. The one is a $2048 \times 2048$ pixels frame lpr31r from an image scanned by the SuperCOSMOS facility at the Royal Observatory of Edinburgh. The other is the a53em frame of $3001 \times 1601$ pixels from an image digitized by a PDS 1010A microdensitometer at the Trieste Observatory. Experts detected spectra in these frames using standard methods of detection on direct and spectral plates.

Applying the DETSP procedure, we had 553 detections (Fig. 2) and 13 undetected spectra (error $<2.5 \%$ ) for the frame lpr31r. For the frame a53em, we had 427 detections (Fig. 3) and 17 undetected spectra (error $<4 \%$ ). A careful analysis showed that the missed spectra are in majority too faint, so they are unusable for further processing (e.g. automated classification). Applying the described method, a very significant number of spectra were automatically detected (True-Positive detections $>96 \%$ ). It is worth to point out that despite the presence of Mtype and Carbon star spectra on the frames, there were no false detections. In both cases, the processing took about $20 \mathrm{~min}$ without displaying supporting plots and images and about twice more with them. Implementation of this MIDAS procedure in a $\mathrm{C}$ language program will significantly reduce this processing time. The results are very encouraging for this procedure as well as for the next steps, extraction and classification, of the OBJPR context for automated processing of objective prism spectra.

Acknowledgements. This research has been supported by a grant from the General Secretariat of Research and Technology of Greece, PENED programme. The authors are grateful to the UK Schmidt Telescope Plate Library (ROE) for the loan of the observational material. 


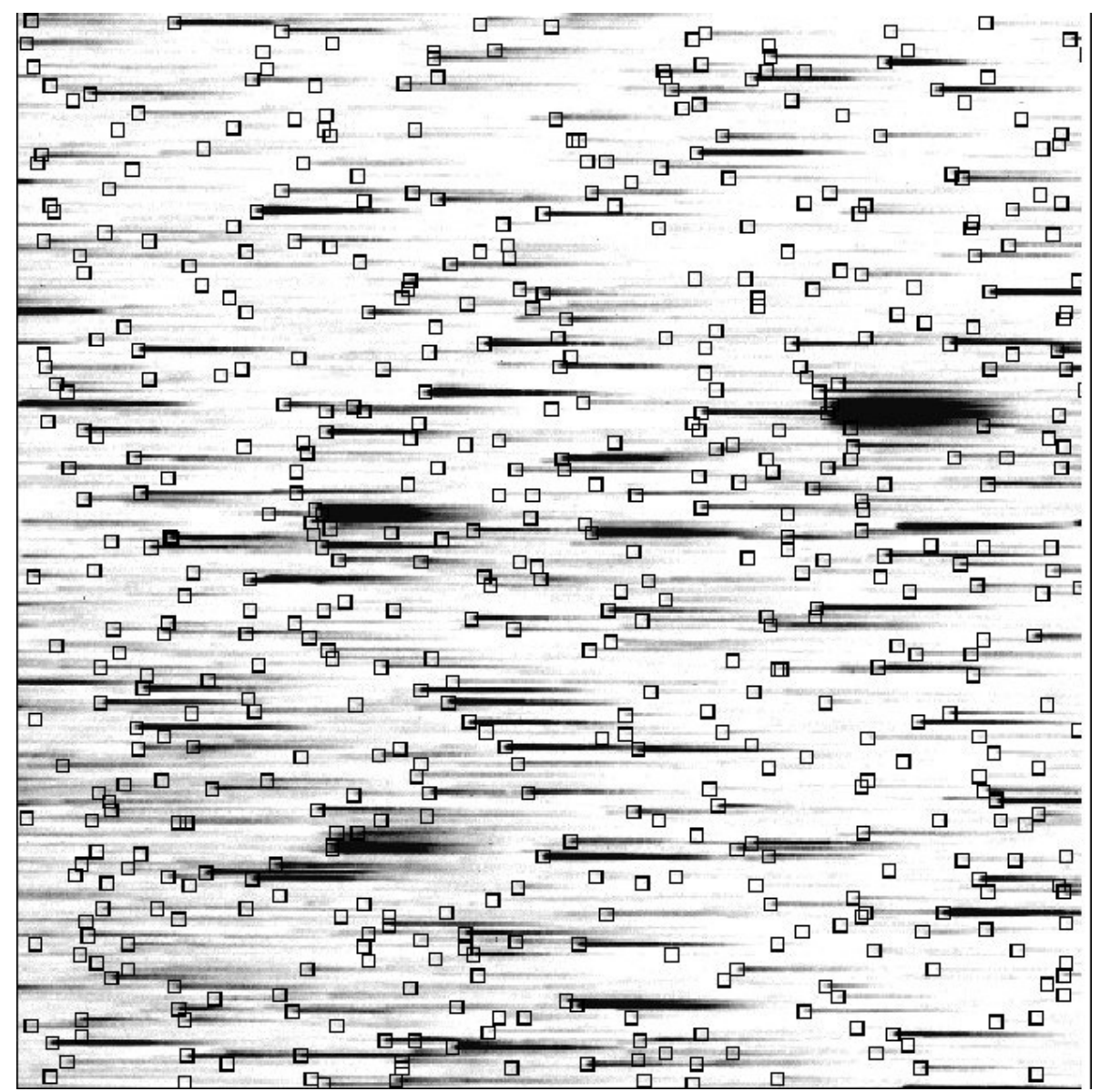

Fig. 2. DETSP test - lp31r frame

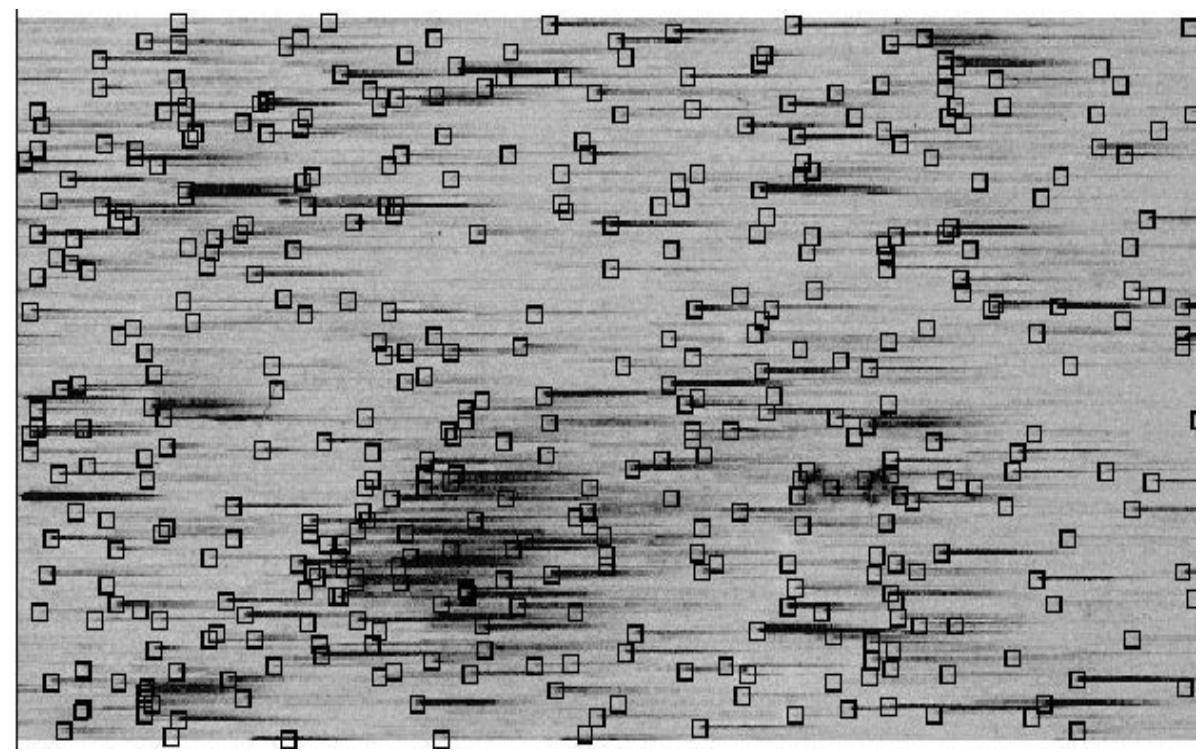

Fig. 3. DETSP test - a53em frame 


\section{References}

Bratsolis E., 1997, in Wide-Field Spectroscopy, E. Kontizas et al. (eds.). Dordrecht: Kluwer Academic Press, p. 119

Hewett P.C., Irwin M.J., Bunclark P., Bridgeland M.T., Kibblewhite E.J., He X.T., Smith M.G., 1985, MNRAS 213, 971

Hewett P.C., Foltz C.B., Chaffee F.H., 1995, AJ 109, 1498

Lahav O., Naim A., Sordé L., Storrie-Lombardi M.C., 1996, MNRAS 283, 207

MIDAS 96NOV, 1996, ESO, Science Data Analysis Group,
Garching, München

Pasian F., Smareglia R., Kontizas E., 1997, in Wide-Field Spectroscopy, E. Kontizas et al. (eds.). Dordrecht: Kluwer Academic Press, p. 123

Schuecker P., 1996, MNRAS 279, 1057

Smareglia R., Pasian F., Kontizas M., Kontizas E., Dapergolas A., 1994, VA 38, 309

Tucholke H.J., Schuecker P., 1992, PASP 104, 704

von Hippel T., Storrie-Lombardi L.J., Storrie-Lombardi M.C., Irwin M.J, 1994, MNRAS 269, 97 